\title{
Typical properties of optimal growth in the Von Neumann expanding model for large random economies
}

\author{
A De Martino† and M Marsilił \\ †INFM-SMC and Dipartimento di Fisica, Università di Roma "La Sapienza", p.le A \\ Moro 2, 00185 Roma (Italy) \\ ‡The Abdus Salam ICTP, Strada Costiera 11, 341014 Trieste (Italy) \\ E-mail: andrea.demartino@roma1.infn.it, marsili@ictp.it
}

\begin{abstract}
We calculate the optimal solutions of the fully heterogeneous Von Neumann expansion problem with $N$ processes and $P$ goods in the limit $N \rightarrow \infty$. This model provides an elementary description of the growth of a production economy in the long run. The system turns from a contracting to an expanding phase as $N$ increases beyond $P$. The solution is characterized by a universal behavior, independent of the parameters of the disorder statistics. Associating technological innovation to an increase of $N$, we find that while such an increase has a large positive impact on long term growth when $N \ll P$, its effect on technologically advanced economies $(N \gg P)$ is very weak.
\end{abstract}

\section{Introduction}

The dynamics in many complex systems involves a flux through components arranged in a heterogeneous network. Examples range from cell metabolism [1], food webs [2], supply networks [3], and river networks [4], to the way in which raw materials are combined and transformed into intermediate or consumption goods in an economy [5]. Mathematically, these phenomena can be cast into linear programming problems, for solving which efficient algorithms exist even for large instances. In each of these cases, however, it is important to understand whether the emerging global properties are due to the specific wiring of the input-output relationships or whether they are generic of large random realizations of any such problem. Put differently, the study of the typical properties of large random systems provides a useful "null hypothesis" against which specific results should be compared. The theoretical machinery for this kind of study is provided by the statistical mechanics of disordered systems and has already been applied to specific large random linear programming problems in the past $[6,7]$.

One of the areas where such problems arise more frequently is economics. The study of typical properties of large random economies $[8,9]$ is particularly relevant, first because it allows one to go beyond the simplistic framework of the so-called 'representative 
agent', by accounting for the heterogeneity across agents and their interactions in many dimensions (technological capabilities, budgets, endowments, etc). Secondly because, contrary to biology, economic time scales are such that evolutionary design might not play a dominant role in shaping the (global properties of) interaction network. Hence, in real economies the latter might be closer to a large random instance than for e.g. metabolic networks.

In this paper, we will study a simple model of economic growth, put forward by J. Von Neumann in the 1930's [5]. It describes economic growth as an autocatalytic process by which the outputs generated at any time are used either for consumption or as inputs for production at the later stage. This model has been widely studied and plays a central role in economic theory [10-13], as it forms the backbone of more refined models providing the key insights for understanding growth in the long runł. Our aim is that of characterizing the growth properties in terms of the underlying structural complexity of the production activity. More precisely, we shall compute the maximal growth rate of the economy as a function of the ratio between the number of production processes and the number of goods, and of the parameters of the distribution of inputoutput matrices. We shall also compute the number of active production processes and of "intermediate" goods, whose output is used entirely for further production. These results shed light on the way in which growth is affected by technological innovation, namely by an enrichment of the repertoire of available technologies, and in turn how this affects the activity levels.

In the rest of the paper, we shall first introduce the model, then present the statistical mechanics approach and finally discuss the results.

\section{The model}

In somewhat simplified terms, Von Neumann's expanding model may be presented as follows. One considers an economy with $P$ commodities (labeled $\mu$ ) and $N$ technologies (labeled $i$ ), each of which can be operated at a non-negative scale $S_{i} \geq 0$ and is characterized by an output vector $\boldsymbol{a}_{i}=\left\{a_{i}^{\mu}\right\}$ and by an input vector $\boldsymbol{b}_{i}=\left\{b_{i}^{\mu}\right\}$, such that $S_{i} a_{i}^{\mu}$ (respectively $S_{i} b_{i}^{\mu}$ ) denotes the units of commodity $\mu$ produced (respectively used) by process $i$ when run at scale $S_{i}$. It is assumed that input/output vectors are fixed in time and that operation scales are the degrees of freedom to be set, for instance, by firms. At time (or period) $t$, the economy is characterized by an aggregate input and output vector for each commodity, $I^{\mu}(t)=\sum_{i} S_{i}(t) b_{i}^{\mu}$ and $O^{\mu}(t)=\sum_{i} S_{i}(t) a_{i}^{\mu}$ respectively. Part of the latter will be used as the input at period $t+1$ whereas the rest, namely

$$
C^{\mu}(t) \equiv O^{\mu}(t)-I^{\mu}(t+1)
$$

$\ddagger$ Models of economic growth address the issue of maximizing discounted welfare over the evolution paths. Turnpike theorems [14] show that optimal paths overlap significantly with the paths of maximal expansion described by Von Neumann's model. 
is consumed at time $t$. In absence of external sources, a necessary condition is that inputs at any time do not exceed the outputs at the previous time, i.e. one must have $C^{\mu}(t) \geq 0$ for all $\mu$ at all times. In this way, the model describes a closed economy which is able to provide the society with all commodities without relying on external sources. Modern economic growth theories introduce a value for the stream of consumption $C^{\mu}(t)$ for all $t$ and $\mu$ - usually by postulating a utility function and a discount factor - and look for optimal growth paths $\left\{S_{i}(t), t \geq 0\right\}_{i=1}^{N}$, as a function of some initial condition $I^{\mu}(0)$. Von Neumann's model instead focuses on the simpler issue of studying the feasibility of paths with a constant rate - i.e. such that $I^{\mu}(t+1)=\rho I^{\mu}(t)$ with $\rho>0$ a constant and, in particular, on computing the highest feasible growth rate. These two problems are related because, under generic conditions, the optimal path coincides with that of maximal expansion apart from an initial transient [14]. This is why Von Neumann's model is relevant for long run properties of models of economic growth. For paths with constant expansion rate, the scales of production have the form $S_{i}(t)=s_{i} \rho^{t}$ and likewise $C^{\mu}(t)=c^{\mu} \rho^{t}$. The (technological) expansion problem then amounts to calculating the maximum $\rho>0$ such that a configuration $s=\left\{s_{i} \geq 0\right\}$ satisfying the condition

$$
c^{\mu} \equiv \sum_{i} s_{i}\left(a_{i}^{\mu}-\rho b_{i}^{\mu}\right) \geq 0 \quad \forall \mu
$$

exists $[15,16]$. In such a configuration the aggregate output of each commodity is at least $\rho$ times its aggregate input. If the maximum $\rho$, which we denote by $\rho^{\star}$, is larger than 1 the economy is 'expanding', whereas it is 'contracting' for $\rho^{\star}<1$. It is a rigorously established fact that if $a_{i}^{\mu} \geq 0$ and $b_{i}^{\mu} \geq 0$ for all $i$ and $\mu, \rho^{\star}$ exists (see [15] for a simple proof). On the other hand, the actual value of $\rho^{\star}$ is expected to depend on the input and output matrices. Intuitively, $\rho^{\star}$ should increase with the number $N$ of technologies and decrease when the economy is required to produce a larger number $P$ of goods.

\section{Statistical mechanics of large random instances}

In this work we study a fully heterogeneous version of above problem with technologies different from each other. We focus on random instances where $a_{i}^{\mu}$ and $b_{i}^{\mu}$ are quenched random variables drawn from a certain probability distribution. More specifically, we shall consider the pair $\left(a_{i}^{\mu}, b_{i}^{\mu}\right)$ as independent and identically distributed for each $i$

and $\mu$. To begin with, let us first simplify the problem by writing $a_{i}^{\mu}=\bar{a}\left(1+\alpha_{i}^{\mu}\right)$ and $b_{i}^{\mu}=\bar{b}\left(1+\beta_{i}^{\mu}\right)$, where $\bar{a}$ and $\bar{b}$ are positive constants while $\alpha_{i}^{\mu}$, $\beta_{i}^{\mu}$ are zero-average quenched random variables. Inserting these into (2) one easily sees that to leading order (in $N$ ) $\rho^{\star}$ is given by the ratio $\bar{a} / \bar{b}$ of the average output and average input coefficients. In particular, the leading part of $\rho$ does not depend on the structure of the input output matrices. The non trivial aspects of the problem are related to the corrections to the leading part. We therefore write the growth rate as

$$
\rho=\frac{\bar{a}}{\bar{b}}\left(1+\frac{g}{\sqrt{N}}\right)
$$


so that (2) becomes

$$
c^{\mu}=\bar{a} \sum_{i} s_{i}\left[\alpha_{i}^{\mu}-\frac{g}{\sqrt{N}}-\left(1+\frac{g}{\sqrt{N}}\right) \beta_{i}^{\mu}\right] \geq 0 \quad \forall \mu
$$

The problem thus reduces to that of finding the largest value $g^{\star}$ of $g$ for which it is possible to find coefficients $\left\{s_{i} \geq 0\right\}$ satisfying (4).

This issue can be tackled in the limit $N \rightarrow \infty$ employing a standard technique originally due to Gardner [17], which allows to derive the behavior of $g^{\star}$ as a function of the control parameter $n=\lim _{N \rightarrow \infty} N / P$. The volume of configuration space occupied by micro-states satisfying (2) at fixed disorder is given by

$$
V_{\boldsymbol{\alpha}, \boldsymbol{\beta}}(g)=\int_{0}^{\infty} d \boldsymbol{s} \prod_{\mu} \theta\left[\frac{1}{\sqrt{N}} \sum_{i} s_{i}\left[\alpha_{i}^{\mu}-\frac{g}{\sqrt{N}}-\left(1+\frac{g}{\sqrt{N}}\right) \beta_{i}^{\mu}\right]\right] \delta\left(\sum_{i} s_{i}-N\right)
$$

where we introduced a linear constraint $\sum_{i} s_{i}=N$. The typical volume occupied by solutions for $N \rightarrow \infty$ reads instead

$$
V_{\text {typ }}(g) \sim e^{N v_{\text {typ }}(g)}
$$

where

$$
v_{\text {typ }}(g)=\lim _{N \rightarrow \infty} \frac{1}{N} \overline{\log V_{\boldsymbol{\alpha}, \boldsymbol{\beta}}(g)}=\lim _{r \rightarrow 0} \lim _{N \rightarrow \infty} \frac{1}{N r} \log \overline{\left[V_{\boldsymbol{\alpha}, \boldsymbol{\beta}}(g)\right]^{r}}
$$

where the last equality contains the replica trick and the over-bar stands for an average over the quenched disorder, that is over the vectors $\alpha_{i}^{\mu}$ and $\beta_{i}^{\mu}$. As usual, the leading contributions to $v_{\text {typ }}(g)$ in the limit $N \rightarrow \infty$ come from the first two moments of the distribution of $\alpha_{i}^{\mu}$ and $\beta_{i}^{\mu}$. Given that $\alpha_{i}^{\mu}$ and $\beta_{i}^{\mu}$ have zero mean, the only property of the disorder distribution which enters the final result is the covariance matrix of the disorder. Actually, the explicit calculation shows that $g^{\star}$ only depends on the parameter

$$
k=\overline{\left(\alpha_{i}^{\mu}-\beta_{i}^{\mu}\right)^{2}}
$$

To make a concrete example, consider the input (output) matrices where $b_{i}^{\mu}=b$ $\left(a_{i}^{\mu}=a\right)$ for $B$ values of $\mu$ and $b_{i}^{\mu}=0\left(a_{i}^{\mu}=0\right)$ otherwise. Then it is easy to see that $\bar{b}=b B / N(\bar{a}=a A / N), \overline{\left(\beta_{i}^{\mu}\right)^{2}}=N / B-1\left(\overline{\left(\alpha_{i}^{\mu}\right)^{2}}=N / A-1\right)$ and $\overline{\alpha_{i}^{\mu} \beta_{i}^{\mu}}=0$ so that $k=N / A+N / B-2$. In particular, the case where outputs and inputs are few corresponds to $k \gg 1$.

After expressing the $\theta$-functions via their integral representations and carrying out the disorder average one finds

$$
\overline{\left[V_{\boldsymbol{\alpha}, \boldsymbol{\beta}}(g)\right]^{r}}=\int J_{1}(\boldsymbol{q}) J_{2}(\boldsymbol{q}) d \boldsymbol{q}
$$

where $\boldsymbol{q}$ is a vector of order parameters $\left\{q_{\ell \ell^{\prime}}\right\}_{\ell<\ell^{\prime}}^{1, r}$ representing the overlaps between the configurations in different replicas, $q_{\ell \ell^{\prime}}=(1 / N) \sum_{i} s_{i \ell} s_{i \ell^{\prime}}$, and

$$
\begin{aligned}
& J_{1}(\boldsymbol{q})=\int_{0}^{\infty} D \boldsymbol{c} \int_{-\infty}^{\infty} D \boldsymbol{z} \prod_{\mu} e^{i \sum_{\ell} z_{\ell}^{\mu}\left(c_{\ell}^{\mu}+g\right)-\frac{k}{2} \sum_{\ell, \ell^{\prime}} q_{\ell \ell^{\prime}} z_{\ell}^{\mu} z_{\ell^{\prime}}^{\mu}} \\
& J_{2}(\boldsymbol{q})=\int_{0}^{\infty} D \boldsymbol{s} \prod_{\ell} \delta\left(\sum_{i} s_{i \ell}-N\right) \prod_{\ell \leq \ell^{\prime}} \delta\left(\sum_{i} s_{i \ell} s_{i \ell^{\prime}}-N q_{\ell \ell^{\prime}}\right)
\end{aligned}
$$


Von Neumann's model for large random economies

$$
=\int d \boldsymbol{R} d \boldsymbol{m} \int_{0}^{\infty} D s e^{-\sum_{\ell} m_{\ell}\left(\sum_{i} s_{i \ell}-N\right)-\sum_{\ell \leq \ell^{\prime}} R_{\ell \ell^{\prime}}\left(\sum_{i} s_{i \ell} s_{i \ell^{\prime}}-N q_{\ell \ell^{\prime}}\right)}
$$

The space of solutions $\left\{s_{i}\right\}$ is a convex set, hence we expect the replica-symmetric approximation to be exact in this case. We therefore evaluate (9) and (10) imposing the Ansatz

$$
q_{\ell \ell^{\prime}}=q+\chi \delta_{\ell \ell^{\prime}}, \quad R_{\ell \ell^{\prime}}=\frac{\beta+\tau^{2}}{2} \delta_{\ell \ell^{\prime}}-\tau^{2}, \quad m_{\ell}=m .
$$

Putting things together, Eq. (6) yields

$$
v_{\text {typ }}(g)=\operatorname{extr}_{q, \chi}\left[F_{1}(q, \chi)+\operatorname{extr}_{\beta, \tau, m} F_{2}(q, \chi, \beta, \tau, m)\right]
$$

where $\operatorname{extr}_{x} f(x)$ denotes the operation of taking the extremum of $f(x)$, and

$$
\begin{aligned}
F_{1} & =\frac{1}{n}\left\langle\log \int_{0}^{\infty} \frac{d c}{\sqrt{2 \pi k \chi}} e^{-\frac{(c+g+\xi \sqrt{k q})^{2}}{2 k \chi}}\right\rangle_{\xi} \\
& =\frac{1}{n}\left\langle\log \left[\frac{1}{2} \operatorname{erfc} \frac{g+\xi \sqrt{q k}}{\sqrt{2 k \chi}}\right]\right\rangle_{\xi} \\
F_{2} & =m+\frac{1}{2} \beta(\chi+q)-\frac{1}{2} \chi \tau^{2}+\left\langle\log \int_{0}^{\infty} d s e^{-(m+\xi \tau) s-\beta s^{2} / 2}\right\rangle_{\xi}
\end{aligned}
$$

The brackets $\langle\ldots\rangle_{\xi}=\int_{-\infty}^{\infty} \frac{d \xi}{\sqrt{2 \pi}} e^{-\xi^{2} / 2} \ldots$ stand for an average over the unit variance Gaussian random variable $\xi$. The problem can in principle be solved in a straightforward way, by analyzing the saddle point equations for any value of $g$. We expect, however that as $g \rightarrow g^{\star}$ the typical volume $V_{\text {typ }}$ shrinks until just one solution remains (modulo re-scalings of the $s_{i}$ 's). Hence $\chi$, which describes the fluctuation $s_{i}$ among feasible solutions, should also vanish as $g \rightarrow g^{\star}$. Hence the conditions $g=g^{\star}$ and $\chi=0$ are equivalent. When $\chi \rightarrow 0$ the integral in (13) can be calculated by steepest descent. The distribution of $c^{\mu}$ in Eq. (4) can be read off Eq. (13) and it has the form

$$
p(c)=\phi_{0} \delta(c)+\theta(c) e^{-\frac{\left(c+g^{\star}\right)^{2}}{2 q k}}
$$

where

$$
\phi_{0}=\frac{1}{2}\left(1+\operatorname{erf} \frac{g^{\star}}{\sqrt{2 q k}}\right)
$$

is the fraction of commodities that are not consumed (namely for which $c^{\mu}=0$ ) at $g=g^{\star}$. These commodities are uniquely used as intermediate goods in the production process. A further important observation is that $F_{1}$ and hence $v_{\text {typ }}$ depend on $g^{\star}$ only through the combination $g^{\star} / \sqrt{k}$. This completely characterizes the dependence of the maximal growth rate on the parameters of the disorder distribution.

If as $g \rightarrow g^{\star}$ the volume of feasible solutions shrinks to zero, the integral on $s$ in $F_{2}$ must ultimately be dominated by a single value. This is consistent with a $1 / \chi$ divergence of the parameters $\beta, \tau$ and $m$. Hence it is convenient to introduce the variables

$$
b=\chi \beta, \quad t=\chi \tau, \quad z=-m / \tau
$$


which remain finite in the limit $\chi \rightarrow 0$ and turn out (after some algebra) to be given by the solution of

$$
\begin{aligned}
q & =\frac{\left\langle(z-\xi)^{2} \theta(z-\xi)\right\rangle_{\xi}}{\langle(z-\xi) \theta(z-\xi)\rangle_{\xi}^{2}} \\
b & =-\langle\xi(z-\xi) \theta(z-\xi)\rangle_{\xi} \\
t & =-\frac{\langle\xi(z-\xi) \theta(z-\xi)\rangle_{\xi}}{\langle(z-\xi) \theta(z-\xi)\rangle_{\xi}}
\end{aligned}
$$

In analogy with what we did for $c^{\mu}$, it is possible to compute the distribution of $s_{i}$. In particular the fraction of inactive technologies $\left(s_{i}=0\right)$ is found to be

$$
\psi_{0}=\frac{1}{2} \operatorname{erfc}\left(\frac{z}{\sqrt{2}}\right) .
$$

Eqs. (18) can be solved numerically to the desired accuracy to yield $b, t$ and $z$ as a function of $q$. We are left with two saddle point conditions, for $q$ and $\chi$, respectively. The latter reads

$$
t^{2}=\frac{q}{n}\left\langle(\xi+g / \sqrt{k q})^{2} \theta(\xi+g / \sqrt{k q})\right\rangle_{\xi}
$$

The saddle point equation for $q$ requires a bit more work. Indeed for $\chi \rightarrow 0$ the leading contributions of $F_{1}$ and $F_{2}$ are of order $1 / \chi$ but they cancel exactly. One must therefore consider the next-to-leading-order correction, of order $\log \chi$. We note, in passing, that this is consistent with the volume of solutions vanishing as $v_{\text {typ }}(g) \sim \chi^{\gamma N}$ as $g \rightarrow g^{\star}$. After some algebra, the final equation takes the particularly simple form

$$
\phi_{0}=n\left(1-\psi_{0}\right) \text {. }
$$

This condition implies that the number of active processes equals that of intermediate commodities at $g^{\star}$. Noting that for any $\mu$ such that $c^{\mu}=0$ we have a linear equation for the scales $s_{i}>0$, we see also that (21) simply corresponds to the requirement that the number of equations should match the number of variables.

Eqs. (18), (20) and (21) allow us to compute $g^{\star}$ as a function of $n$ and $k$. As already noticed, the dependence $g^{\star} \propto \sqrt{k}$ can be read off directly from the equations, hence $g^{\star} / \sqrt{k}$ is an universal function of $n$, independent of the details of the distribution of input-output coefficients (as long as they are i.i.d.). Actually, since $k$ enters the equations only through the combination $g^{\star} / \sqrt{k}$, and this is a function of $n$, then the solution itself will be universal, i.e. independent of $k$.

Fig. 1 reports the rescaled growth rate $g^{\star} / \sqrt{k n}$ as a function of $n$. This line separates the region of feasible solutions with growth rates $g \leq g^{\star}$ from the region of unfeasible solutions. $g^{\star}$ crosses the line $g^{\star}=0$ at $n=1$, as can be checked explicitly. Indeed, by Eq. (16), $g^{\star}=0$ corresponds to a situation where half of the goods are not consumed $\left(\phi_{0}=1 / 2\right)$ while Eq. (20) yields $t^{2}=q /(2 n)$, which is consistent with the other equations for $z=0$, i.e. $n=1$. At this same point, half of the technologies are operated $\left(\psi_{0}=1 / 2\right)$. This means that, when there are more technologies than goods 


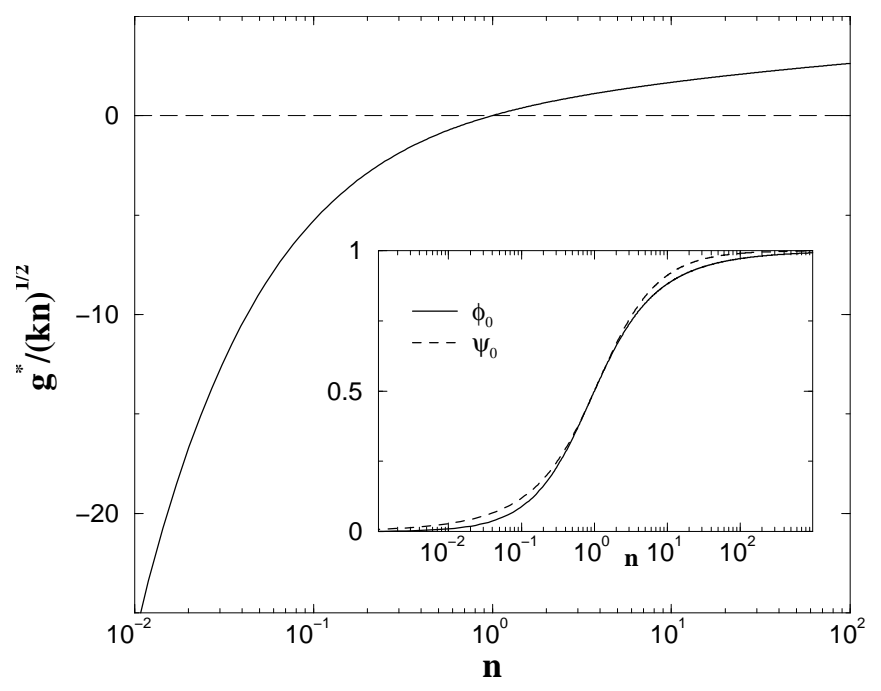

Figure 1. Behavior of $g^{\star} / \sqrt{k n}$ vs $n$. Inset: $\phi_{0}$ and $\psi_{0}$ (related by (21)) vs $n$.

$(n>1)$ a growth rate higher than that of the ratio $\bar{a} / \bar{b}$ of average output and input coefficients is achievable. The growth rate is instead smaller than $\bar{a} / \bar{b}$ when $n<1$.

The inset of Fig. 1 shows the fraction of inactive processes $\psi_{0}$ and that of intermediate commodities $\phi_{0}$ at $g^{\star}$, as a function of $n$. For what we said earlier, these are universal functions of $n$ independent of the details of the disorder distribution. Both $\phi_{0}$ and $\psi_{0}$ tend to one when $n$ increases.

\section{Discussion}

In summary, we have studied the typical properties of the Von Neumann expanding model in the case where input-output matrices have i.i.d. random elements. We characterize the region of feasible expansion paths and focus on the solutions at its boundary, which correspond to the paths of maximal expansion. We uncover an universal behavior, independent of the details of the distribution, of the relevant quantities. In particular we find that as the number $N$ of technologies grows, the optimal growth rate increases, but the economy becomes more and more selective both on the processes which are used and on the goods which are consumed.

At a purely speculative level, our results allow us to draw conclusions on how long term growth on the maximal expansion path will be affected by technological innovation. The latter, defined as the introduction of new designs, i.e. new feasible ways of combining inputs to produce desirable outputs [18] would just correspond, in our simplified world, to an increase in the number $N$ of transformation processes which the economy has at its disposal. By Eq. (3) the change in the growth rate is related to the change in $g^{\star} / \sqrt{n}$, which is plotted in Fig. 1. This shows that when $n$ is small 
$(n \ll 1)$

$$
\delta \rho \propto \frac{\delta n}{n^{3 / 2} \sqrt{P}}
$$

i.e. an increase in $N$ can have a large positive impact on long term growth. For technologically mature economies $(n \gg 1)$ instead, $g^{\star} / \sqrt{n}$ increases much more slowly, hence technological innovation has much smaller effect on long term growth. These insights are remarkably similar to those derived in [9] for model of general economic equilibrium.

There are several directions in which the present work could be extended. First, it would be desirable to study more realistic ensembles of input-output matrices, or more detailed models of economic growth. In particular, realistic technologies only have a finite number of inputs and outputs, which would call for the use of techniques for disordered systems with diluted interactions. It would also be interesting to generalize this study to the case where the input-output transformation is subject to stochasticity. A further natural extension of this approach concerns the analysis of typical properties of large random metabolic [1] or supply networks [3], or of food webs [2].

\section{References}

[1] Segrè D, Vitkup D and Church GM 2002 Proc. Nat. Acad. Sci. 9915112

[2] Cohen JE, Briand F and Newman CM 1990 Community Food Webs: Data and Theory (Springer, Berlin)

[3] Helbing D, Lämmer S, Seidel T, Šeba P and Platkowski T 2004 Phys. Rev. E 70066116

[4] Rodriguez-Iturbe I and Rinaldo A 1996 Fractal River Basins: Chance and Self-Organization (Cambridge University Press, Cambridge, UK)

[5] Von Neumann J 1937 Ergebn. eines Math. Kolloq. 8. English translation: Von Neumann J 1945 Rev. Econ. Studies 131

[6] Korutcheva E, Opper M and Lopez B 1994 J. Phys. A: Math. Gen 27 L645

[7] Nishimori H 2004 Statistical physics of spin glasses and information processing (Oxford University Press, Oxford, UK)

[8] Föllmer H 1974 J. Math. Econ. 151

[9] De Martino A, Marsili M and Perez Castillo I 2004 J. Stat. Mech. P04002

[10] Champernowne DG 1945 Rev. Econ. St. 1310

[11] Gale D 1956 In Kuhn HW and Tucker AW (Eds), Linear inequalities and related systems (Ann. Math. Studies 38, Princeton, NJ)

[12] Kemeny JG, Morgenstern O and Thompson GL 1956 Econometrica 24115

[13] Morgenstern O and Thompson GL 1976 Mathematical theory of expanding and contracting economies (Heath-Lexington, Boston, MA)

[14] McKenzie LW 1986 "Optimal Economic Growth, Turnpike Theorems and Comparative Dynamics", in Arrow KJ and Intriligator MD (Eds), Handbook of Mathematical Economics, Vol. III (NorthHolland, Amsterdam)

[15] Gale D 1960 The theory of linear economic models (The University of Chicago Press, Chicago, IL)

[16] Thompson GL 1989 In Dore M, Chakravarty S and Goodwin R (Eds), John von Neumann and modern economics (Clarendon Press, Oxford, UK)

[17] Gardner E 1988 J. Phys. A: Math. Gen. 21257

[18] Romer P 1990 J. Pol. Econ. 98 S72 